\title{
Male accessory gland substances from Aedes albopictus affect the locomotor activity of Aedes aegypti females
}

\author{
Tamara Nunes Lima-Camara ${ }^{1}{ }^{+}$, Claudia Torres Codeço ${ }^{1}$, Nildimar Alves Honório², \\ Rafaela Vieira Bruno ${ }^{3,4}$, Alexandre Afranio Peixoto ${ }^{3,4,+}$, Leon Philip Lounibos ${ }^{5}$ \\ 'Programa de Computação Científica ${ }^{2}$ Laboratório de Transmissores de Hematozoários \\ ${ }^{3}$ Laboratório de Biologia Molecular de Insetos, Instituto Oswaldo Cruz-Fiocruz, Rio de Janeiro, RJ, Brasil \\ ${ }^{4}$ Instituto Nacional de Ciência e Tecnologia/Entomologia Molecular, Conselho Nacional de Desenvolvimento Científico e Tecnológico, \\ Brasilia, DF, Brasil ${ }^{5}$ Florida Medical Entomology Laboratory, University of Florida, Vero Beach, Florida, USA
}

\begin{abstract}
Dengue is one of the world's most important mosquito-borne diseases and is usually transmitted by one of two vector species: Aedes aegypti or Aedes albopictus. These two diurnal mosquitoes are frequently found coexisting in similar habitats, enabling interactions between adults, such as cross-mating. The objective of this study was to assess cross-mating between Ae. aegypti females and Ae. albopictus males under artificial conditions and evaluate the locomotor activity of Ae. aegypti virgin females injected with male accessory gland (MAG) homogenates to infer the physiological and behavioural responses to interspecific mating. After seven days of exposure, 3.3-16\% of Ae. aegypti females mated with Ae. albopictus males. Virgin Ae. aegypti females injected with conspecific and heterospecific MAGs showed a general decrease in locomotor activity compared to controls and were refractory to mating with conspecific males. The reduction in diurnal locomotor activity induced by injections of conspecific or heterospecific MAGs is consistent with regulation of female reproductive activities by male substances, which are capable of sterilising female Ae. aegypti through satyrisation by Ae. albopictus.
\end{abstract}

Key words: Aedes aegypti - Aedes albopictus - cross-mating - locomotor activity - laboratory

Dengue is one of the most important mosquito-borne diseases in the world, being endemic in approximately 112 countries, with an estimation of more than 300 million cases per year (Bhatt et al. 2013). The etiologic agent is a RNA Flavivirus, that is typically transmitted to humans by the mosquito Aedes (Stegomyia) aegypti (Linnaeus, 1762). Another vector of dengue of increasing importance worldwide is Aedes (Stegomyia) albopictus (Skuse, 1894) (Consoli \& Lourenço-de-Oliveira 1994).

Originating from Asia, Ae. albopictus invaded the United States of America (USA) and Brazil in the 1980s and, despite its high susceptibility to dengue virus infection under laboratory conditions (Miller \& Ballinger 1988, Lourenço-de-Oliveira et al. 2003, de Castro et al. 2004), it is still considered a potential dengue vector in Brazil, although dengue viruses have been isolated by pools of larvae in nature (Serufo et al. 1993, Degallier et al. 2003, Figueiredo et al. 2010).

Although Ae. aegypti is usually associated with urban areas and Ae. albopictus prefers habitats with more vegetation (Consoli \& Lourenço-de-Oliveira 1994, Braks et al. 2003, Lima-Camara et al. 2006, Rey et al.

doi: 10.1590/0074-0276130381

Financial support: FAPERJ (E-26/111.302/2011), INCT-EM/CNPq (407744/2012-6), NIH-USA (R21 AI095780)

+ In memoriam

+ Corresponding author: tammylimacamara@gmail.com

Received 25 July 2013

Accepted 26 September 2013
2006), these species may be sympatric in urban and suburban areas, where they may coexist in the same larval habitats (Braks et al. 2003, Honório et al. 2009). In addition, Ae. aegypti and Ae. albopictus adults frequently show bimodal diurnal activity periods under natural and artificial conditions, flying, mating and blood-feeding preferentially during the morning and afternoon hours (Hartberg 1971, Gubler \& Bhattacharya 1972, LimaCamara 2010).

The invasion of Ae. albopictus in the USA in the 1980s led to declines in distribution and abundance of Ae. aegypti in the southeast of that country (Lounibos 2002). Among the explanations proposed to explain the displacement of Ae. aegypti populations by Ae. albopictus, the most commonly cited is asymmetric interspecific larval competition, which usually favours $A e$. albopictus, under conditions of limited resources commonly encountered in their container habitats (Juliano 1998, Daugherty et al. 2000, Braks et al. 2004).

Nevertheless, in areas where Ae. aegypti and Ae. albopictus are sympatric, these species swarm and mate during the same morning and afternoon peaks of activity (Nasci et al. 1989, Honório et al. 2009), which could facilitate asymmetric mating interference between them, a phenomenon called satyrisation (Ribeiro \& Spielman 1986, Ribeiro 1988, Tripet et al. 2011). Ae. aegypti and Ae. albopictus females are usually inseminated only once by males of their own species, because accessory gland (AG) substances transferred from males make mated females refractory to subsequent inseminations (Craig Jr 1967). Thus, cross-mating interference could sterilise a female that cannot produce viable offspring (Leahy \& Craig Jr 1967). 
Satyrisation between these species has been documented under laboratory conditions (Nazni et al. 2009) and in the field (Nasci et al. 1989, Tripet et al. 2011). Under laboratory conditions, Nazni et al. (2009) reported successful bidirectional cross-mating between Ae. albopictus and Ae. aegypti, which was followed by oviposition of sterile eggs. Results of cage experiments by Nasci et al. (1989) and Bargielowski et al. (2013) suggested that Ae. aegypti females are more receptive to insemination by Ae. albopictus males than Ae. albopictus females are receptive to insemination by Ae. aegypti males. In addition, implants of heterologous male AGs (MAGs) resulted in oviposition of sterile eggs by Ae. aegypti females, but no oviposition by Ae. albopictus females (Leahy \& Craig Jr 1965). Tripet et al. (2011) also injected heterologous MAG in Ae. aegypti and Ae. albopictus virgin females and reported sterilisation for the former species, but no effect on the ability of Ae. albopictus to mate with their own species. Tripet et al. (2011) therefore proposed asymmetric mating interference as a potential neglected explanation for the observed displacement of Ae. aegypti populations in areas where Ae. albopictus invaded, such as the southeastern USA and Bermuda (Lounibos 2007, Kaplan et al. 2010).

Locomotor activity analysis of mosquitoes can be useful for testing the effect of external or internal factors, such as dengue infection on adult female performance (Lima-Camara et al. 2011). Moreover, previous studies have shown that mating has a significant effect on flight activity of many mosquito species, including Ae. aegypti (Clements 1999). Indeed, the AGs of male mosquitoes may produce substances that are transferred to females during mating and alter their physiology and behaviour. The effects of such substances include the inhibition of female remating, stimulation of oviposition and preoviposition behaviours and the inhibition of hostseeking behaviour (Klowden 1996, 1999).

The objective of this study was to verify the frequency of cross-mating between Ae. aegypti females and Ae. albopictus males under artificial conditions and evaluate the locomotor activity of Ae. aegypti virgin females injected with conspecific vs. heterospecific AGs to infer their physiological and behavioural responses to interspecific mating.

\section{MATERIALS AND METHODS}

Mosquito rearing - Ae. aegypti and Ae. albopictus eggs were obtained from colonies at Laboratory of Transmitters of Hematozoa, at Oswaldo Cruz Foundation, state of Rio de Janeiro (RJ), Brazil. Both Ae. aegypti and Ae. albopictus colonies are maintained with eggs collected from areas of sympatry in RJ since 1987. Larvae were reared in plastic trays with tap water and fish food (Te$\operatorname{tramin}^{\circledR}$ ). Trays were kept in an incubator with photoperiod of light/dark (LD) $12: 12$, at $27^{\circ} \mathrm{C}$ and $80 \%$ relative humidity. Pupae of both species were isolated individually to ensure virgin males and females after emergence.

Dissection of MAGs for injection of Ae. aegypti females - Dissected AGs of 25 virgin four-six day-old Ae. aegypti and 25 Ae. albopictus males (Consoli \&
Lourenço-de-Oliveira 1994) were stored in $50 \mu \mathrm{L}$ of saline. Dissections in saline were made under a stereoscopic microscope. Each injecting solution was prepared with 50 pairs of AGs in $50 \mu \mathrm{L}$ of saline. Prior to injections, this $\mathrm{AG}$ solution was sonicated for $1 \mathrm{~min}$ and centrifuged for $1 \mathrm{~h}$ at $13,000 \mathrm{~g}$.

Five-seven-day-old virgin Ae. aegypti females were individually injected by intrathoracic inoculation either with $0.28 \mu \mathrm{L}$ of saline (control group), $0.28 \mu \mathrm{L}$ of $\mathrm{AG}$ solution of Ae. aegypti males or $0.28 \mu \mathrm{L}$ of AG solution of Ae. albopictus males (MAG groups) using a Nanoject microinjector (Drummond Scientific). Since each solution contained one $\mathrm{AG} / \mu \mathrm{L}$, we injected more than a quarter (0.28) of an AG into each Ae. aegypti female. This dose is sufficient for females to respond as if they have been inseminated, since each MAG apparently contains enough active material to sterilise at least 64 females (Craig Jr 1967).

Analysis of locomotor activity - The activity of Ae. aegypti and Ae. albopictus in treatment and control groups was recorded using a larger version of the Drosophila Activity Monitor (TriKinetics) as described in Lima-Camara et al. (2011). After inoculation with saline or AGs, each Ae. aegypti female was individually placed in a glass tube $(1 \mathrm{~cm} \times 7 \mathrm{~cm})$ with a cotton plug soaked in $15 \%$ sucrose solution and the tube was placed in the Activity Monitor inside a Precision Scientific Model 818 Incubator under a constant temperature of $25^{\circ} \mathrm{C}$ and a photoperiod of $12 \mathrm{~h}$ of light and $12 \mathrm{~h}$ of dark (LD 12:12). For each mosquito, the total locomotor activity during 30 min-intervals was recorded continuously for six days after inoculation. We calculated the William's mean (Wm) as an estimate of the central tendency of activity during each time interval (Gentile et al. 2009, Brito et al. 2013). $\mathrm{Wm}$ is a modified geometric mean that accommodates frequent zero values (Williams 1937). Only data from individuals that lived until the fourth day after inoculation were retained and the data analysis was carried out comparing the activity of control and AG-injected groups from $24 \mathrm{~h}$ (1st day) to $96 \mathrm{~h}$ (4th day) after inoculation. At the end of the locomotor activity experiment, all live Ae. aegypti females of each group were transferred to $17 \mathrm{~cm} \mathrm{x} 17 \mathrm{~cm}$ cages and exposed to conspecific males for $48 \mathrm{~h}$ in a ratio of 1 female: 2 males. After $48 \mathrm{~h}$, all three spermathecae were dissected from live females for detection of Ae. aegypti sperm.

Cross-mating experiment - To assess the frequency of cross-mating between Ae. aegypti females and Ae. albopictus or Ae. aegypti males under conditions in our laboratory, approximately 30 virgin $A e$. aegypti females were placed in a $17 \mathrm{~cm} \times 17 \mathrm{~cm}$ cage, with 40 virgin Ae. aegypti or Ae. albopictus males. Three-five-day-old females and three-five-days-old males were placed together in these cages in two different combinations ( $A e$. aegypti females x Ae. aegypti males and Ae. aegypti females x Ae. albopictus males) for seven days. Each pairing was replicated three times, for a total number of six cages. Cotton soaked in a $15 \%$ sucrose solution was continuously available for all mosquitoes as a sugar source. 
After seven days of exposure to Ae. aegypti or Ae. albopictus males, spermathecae of all live Ae. aegypti females were removed and examined under a compound microscope at 100X magnification.

Statistical analysis - For statistics analysis, we used locomotor activity values of all mosquitoes transformed to $\log +1$. The daily locomotor activity of control and treatment groups was summarised by five indices: (i) the mean total activity, (ii) the mean diurnal activity, (iii) the mean diurnal activity without lights-on, i.e., the mean activity during the photophase except for the first 30 min, which corresponds to the morning peak, (iv) the mean lights-on, which corresponds to the first $30 \mathrm{~min}$ just after the lights-on and (v) the mean nocturnal activity. Two-way ANOVA (in conjunction with Tukey test) was applied to test differences in the locomotor activity, measured by these indices, between control (saline injected) vs. MAG groups (injections with Ae. aegypti male's AG or Ae. albopictus male's AG). The second factor in the two-way ANOVA is "block", since the locomotor study was carried out twice. All statistics were done in R Program version 2.15.1 (R Core Team 2012).

\section{RESULTS}

In both control and treatment groups, Ae. aegypti females showed a bimodal rhythm, with peaks at the lights-on and the lights-off (Figs 1,2). The lights-on peak was higher in the control group whereas, in general, $A e$. aegypti females injected with heterospecific MAG substance showed the highest peak at lights-off (Figs 1, 2). Ae. aegypti females injected with MAGs showed a general decrease in all activity indices compared to controls (Table I), showed by lower arithmetic means.

The total activity of the three tested groups showed a significant effect of status (injected with saline vs. injected with $\mathrm{AG})[\mathrm{F}=10.536$; degree of freedom $(\mathrm{df})=2$; $\mathrm{p}<0.001]$, but no significant block effects $(\mathrm{F}=0.292$; $\mathrm{df}$ $=1 ; \mathrm{p}=0.590)$ or interaction between block and status $(\mathrm{F}=1.699 ; \mathrm{df}=2 ; \mathrm{p}=0.186)$. Tukey test (Fig. 3) showed

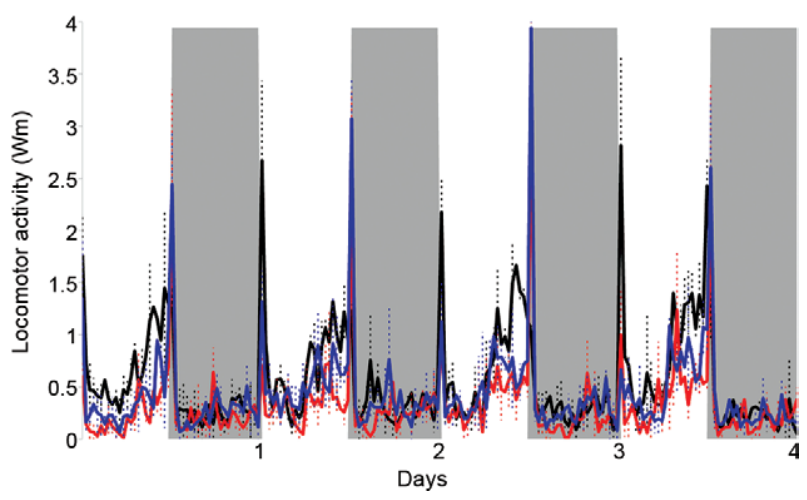

Fig. 1: William's mean (Wm) of locomotor activity of Aedes aegypti females injected with saline (black line-control group), accessory gland (AG) of Ae aegypti males (red line) and AG of Aedes albopictus males (blue line) exposed to four days of $12 \mathrm{~h}$ of light (white columns) and $12 \mathrm{~h}$ of dark (grey columns) (light/dark: 12:12) at $25^{\circ} \mathrm{C}$. Dotted lines represent the standard error of the $\mathrm{Wm}$. a significantly reduced activity of Ae. aegypti virgin females injected with conspecific MAG as compared to the control group $(\mathrm{p}<0.001)$. The total locomotor activity of females injected with Ae. albopictus MAG was lower than the control group as well (Table I), but the difference was not statistically significant $(p=0.156)$, remaining higher than those injected with conspecific MAG (Fig. 3). When the locomotor activity is stratified according to time of day, it becomes evident that only the diurnal behaviour is affected by MAG (Fig. 3). Considering the lights-on peak, that is, the transition between dark and light provides the sharpest discrimination among groups with both MAG-injected groups showing significantly less activity than the control group, with conspecific MAG inducing similar decrease of heterospecific MAG.

At the end of the locomotor activity experiments, live Ae. aegypti females of all the three tested groups were exposed to conspecific males for $48 \mathrm{~h}$. Most Ae. aegypti females injected with saline (control) had positive spermathecae $(84 \%)$, that is, they were inseminated by conspecific males within $48 \mathrm{~h}$. On the other hand, no Ae. aegypti females injected with conspecific MAG were inseminated by conspecific males (Table II). Similar results were observed for Ae. aegypti females injected with Ae. albopictus MAG, among whom 97.3\% (36/37) were unmated after $48 \mathrm{~h}$, despite the heterospecific AG injection (Table II).

Cages 1-3 of the spontaneous mating experiment contained 30 Ae. aegypti females and 40 Ae. aegypti males each and, respectively, 90\% (27/30) and 100\% (27/27) of Ae. aegypti females in cages 1-3 were inseminated by their conspecific males. In cage 2, 35\% (8/23) of Ae. aegypti females were inseminated by their conspecific males.

Cages 4-6 exhibited limited cross-mating between 30 Ae. aegypti females and 40 Ae. albopictus males, but most Ae. aegypti females remained uninseminated after seven days: $96 \%(24 / 25)$ in cage $4,96.7 \%(29 / 30)$ in cage 5 and $83.3 \%(20 / 24)$ in cage 6 (Table III).

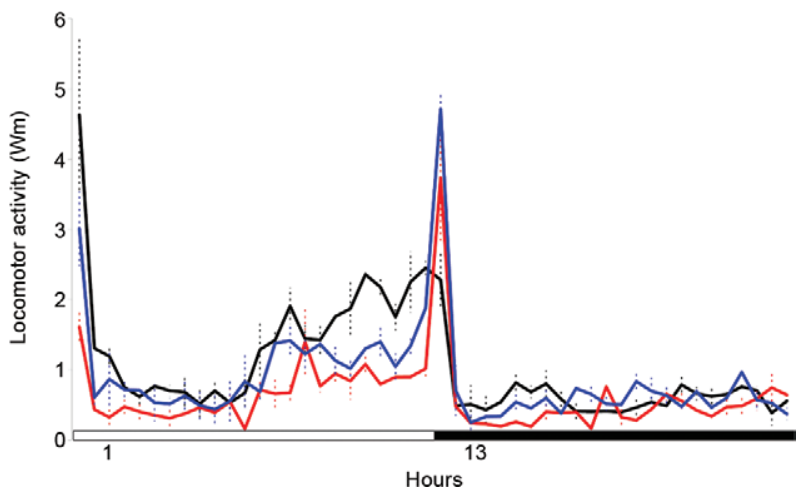

Fig. 2: William's mean (Wm) of locomotor activity of Aedes aegypti females injected with saline (black line-control group), accessory gland (AG) of Ae. aegypti males (red line) and AG of Aedes albopictus males (blue line) under (light dark: 12:12) at $25^{\circ} \mathrm{C}$. Lines represent the 30 min mean activity ( \pm standard error) of control and male AGs injected females in the four tested days. 


\section{DISCUSSION}

In this study, the consequences of mating interactions between two important arboviruses vectors - $A e$. aegypti and Ae. albopictus - were reported. This is the first time that the locomotor activity of Ae. aegypti females injected with saline, conspecific and heterospecific MAG extracts has been evaluated.

Both conspecific and heterospecific MAGs induced a reduction in the intensity of diurnal activity while keeping the overall bimodal pattern, with peaks of activity at lights-on and lights-off. In agreement with our results, Jones (1981) reported similar patterns of flight activity in virgin and inseminated Ae. aegypti females under LD 12:12. Activity reduction associated with MAG injection suggests that females interpreted the injected materials as a signal of insemination. Previous studies suggest that inseminated Aedes females show changes in their activity patterns, because MAGs produce several specific proteins that are transferred to females during mating, influencing reproductive and feeding behaviour (Klowden 1996, 1999, Gillott 2003). Changes in the pattern of activity of inseminated females have also been described for mosquitoes of other genera, such as Anopheles gambiae (Jones \& Gubbins 1977, 1978) and Anopheles stephensi (Rowland 1989).

Activity reduction after heterospecific MAG injection suggests that Ae. albopictus males may induce behavioural changes in Ae. aegypti through mating. However, a few differences were observed between the influences of Ae. aegypti and Ae. albopictus MAGs. The total mean activity of Ae. aegypti injected with $A e$. albopictus MAG extract did not show a significant decrease compared with the control group whereas Ae. aegypti injected with conspecific MAG extract showed a significant decrease. Similar activity reduction in both conspecific and heterospecific injection groups is ob- served during the lights-on peak. These differences may be explained by small differences in MAG composition between species or differences in the female receptor sites. In fact, Ae. aegypti MAG seems to produce several non-homologous proteins that are not found in other Diptera males, such as Culex pipiens, An. gambiae, Drosophila melanogaster (Sirot et al. 2008) and, probably, Ae. albopictus. This deserves future investigations.

Further evidence of the successful mating signal induced by Ae. albopictus MAG in Ae. aegypti females comes from the mating experiments. After exposing saline injected $A$ e. aegypti females to conspecific males for $48 \mathrm{~h}$, most control females successfully mated, while those injected either with Ae. aegypti or Ae. albopictus MAGs remained sperm-negative, indicating that MAG injections from males of either Aedes species were capable of making the Ae. aegypti female refractory to further insemination. Our results agree with Craig Jr (1967) who implanted several Ae. aegypti male tissues in the thorax of virgin Ae. aegypti females and, after 24 $\mathrm{h}$ of recovery, exposed them to conspecific males for an additional $24 \mathrm{~h}$. The author dissected the spermathecae for sperm detection and, as in our study, most (at least 85\%) virgin Ae. aegypti females injected either with saline, testis and gut tissues were inseminated while MAG injected females showed no sign of insemination after 8 $\mathrm{h}$ of exposure to conspecific males. Interestingly, when females were exposed to males immediately after injection, $26 \%$ were inseminated, suggesting that several hours are required before the sterilising effect of MAG is accomplished (Craig Jr 1967).

Shutt et al. (2010) injected virgin Ae. aegypti and anopheline females (An. stephensi and An. gambiae S and $\mathrm{M}$ molecular forms) with their conspecific MAG homogenates and reported a drastic reduction on the likelihood of subsequent mating with conspecific males.

TABLE I

Arithmetic means ( \pm standard error) of total, diurnal, nocturnal, diurnal without lights-on and light-on means of Aedes aegypti females injected with saline, accessory gland (AG) of Ae. aegypti males and AG of Aedes albopictus males

\begin{tabular}{|c|c|c|c|c|}
\hline & $\begin{array}{l}\text { Saline } \\
(n=64)\end{array}$ & $\begin{array}{l}\text { Ae. aegypti male } \mathrm{AG} \\
\qquad(\mathrm{n}=56)\end{array}$ & $\begin{array}{l}\text { Ae. albopictus male AG } \\
\qquad(\mathrm{n}=70)\end{array}$ & $\mathrm{F}$ test $^{a}$ \\
\hline Total mean & $3.01 \pm 0.30$ & $\begin{array}{l}1.75 \pm 0.24 \\
(-41.86)\end{array}$ & $\begin{array}{l}2.63 \pm 0.27 \\
\quad(-12.6)\end{array}$ & $\begin{array}{c}\mathrm{F}=10.536 ; \mathrm{df}=2 ; \\
\mathrm{p}<0.001\end{array}$ \\
\hline Diurnal mean & $3.72 \pm 0.37$ & $\begin{array}{l}2.00 \pm 0.32 \\
(-46.23)\end{array}$ & $\begin{array}{l}3.17 \pm 0.34 \\
(-14.78)\end{array}$ & $\begin{array}{c}\mathrm{F}=18.443 ; \mathrm{df}=2 ; \\
\mathrm{p}<0.001\end{array}$ \\
\hline Nocturnal mean & $2.30 \pm 0.39$ & $\begin{array}{l}1.50 \pm 0.28 \\
(-34.78)\end{array}$ & $\begin{array}{l}2.09 \pm 0.31 \\
(-9.13)\end{array}$ & $\begin{array}{c}\mathrm{F}=1.712 ; \mathrm{df}=2 ; \\
\quad p=0.183\end{array}$ \\
\hline $\begin{array}{l}\text { Diurnal mean } \\
\text { without lights-on }\end{array}$ & $3.49 \pm 0.37$ & $\begin{array}{l}1.87 \pm 0.32 \\
(-46.42)\end{array}$ & $\begin{array}{l}2.97 \pm 0.35 \\
\quad(-14.9)\end{array}$ & $\begin{array}{c}\mathrm{F}=16.519 ; \mathrm{df}=2 ; \\
p<0.001\end{array}$ \\
\hline Light-on mean & $9.06 \pm 1.69$ & $\begin{array}{l}4.84 \pm 1.56 \\
(-46.58)\end{array}$ & $\begin{array}{l}7.64 \pm 1.76 \\
(-15.67)\end{array}$ & $\begin{array}{c}\mathrm{F}=14.702 ; \mathrm{df}=2 ; \\
\mathrm{p}<0.001\end{array}$ \\
\hline
\end{tabular}

$a$ : result of ANOVA testing variation among three groups. Transformed log +1 values were used for statistics. Numbers inside parenthesis represent the percentage of decrease (-) of means of Ae. aegypti females injected with Ae. aegypti and Ae. albopictus AG in relation to the control group (saline). df: degrees of freedom. 
Moreover, the authors cross-injected MAG extracts from $\mathrm{M}$ molecular form from males into $\mathrm{S}$ molecular form females and vice-versa in order to test the divergence in MAG proteins for refractoriness to further mating. These authors found refractoriness for further mating in cross-injected An. gambiae M and S molecular forms, which suggests that these forms have not diverged in relation to sex peptides responsible for female monogamy.

Our data also corroborates the results of Tripet et al. (2011). These authors cross-injected Ae. aegypti and Ae. albopictus females and reported that the majority of $A e$. albopictus females injected with Ae. aegypti MAG subsequently mated with conspecific males. In contrast, $A e$. aegypti and Ae. albopictus females injected with conspecific MAG, as well as Ae. aegypti females injected with Ae. albopictus MAG, were refractory when exposed to conspecific males.

Although multiple reproductive barriers isolating $A e$. aegypti and Ae. albopictus have been identified (Leahy \& Craig Jr 1967), cross-mating between these two species is reported in the field, although at a low frequency (Tripet et al. 2011). Under laboratory conditions in the absence of conspecifics, Bargielowski et al. (2013) reported a high cross-mating frequency $(>30 \%)$ between Ae. aegypti females and Ae. albopictus males when they were exposed for three weeks. In our study, using cage
A

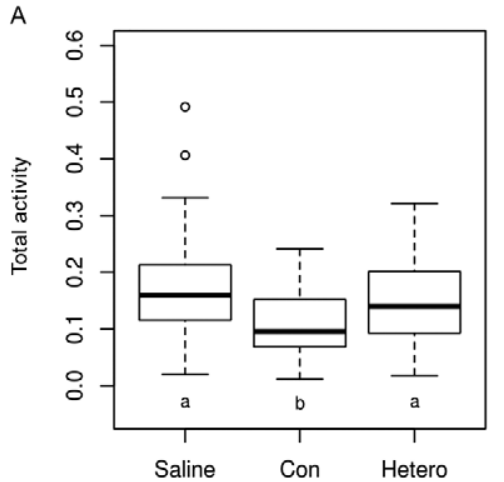

c

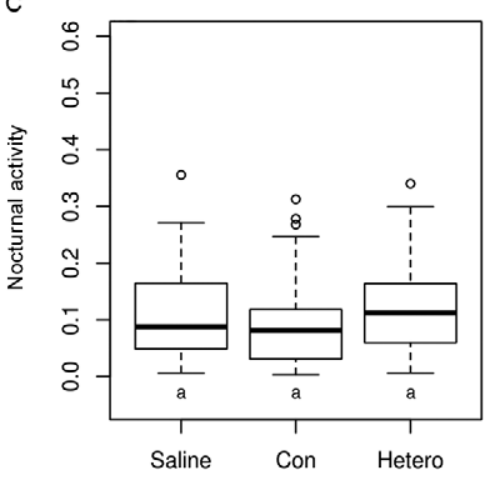

B

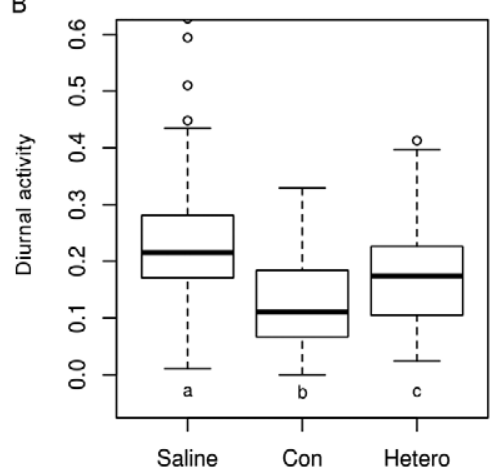

D

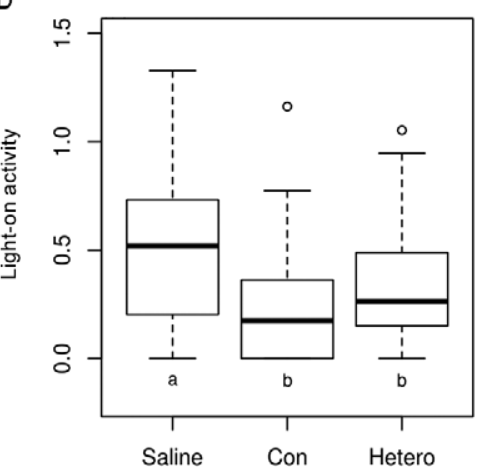

Fig. 3: boxplots of means of Aedes aegypti females injected with saline $(\mathrm{n}=64)$, accessory gland $(\mathrm{AG})$ of Ae. aegypti males $($ Con $)(\mathrm{n}=56)$ and AG of Aedes albopictus males (Hetero) $(\mathrm{n}=70)$. A: total activity (diurnal + nocturnal); B: diurnal activity; C: nocturnal activity; D: lights-on activity. Different letters identify groups that were statistically different $(\mathrm{p}<0.05)$ according to the Tukey test.

TABLE II

Number and percentage of positive and negative dissected spermathecae of Aedes aegypti females injected with saline, accessory glands (AGs) of Ae. aegypti males and AGs of Aedes albopictus males and exposed to conspecific males for two days

\begin{tabular}{lccc}
\hline Injection & $\begin{array}{c}\text { Dissected females } \\
(\mathrm{n})\end{array}$ & $\begin{array}{c}\text { Positive spermathecae } \\
\mathrm{n}(\%)\end{array}$ & \multicolumn{2}{c}{$\begin{array}{c}\text { Negative spermathecae } \\
\mathrm{n}(\%)\end{array}$} \\
\hline Saline (control) & 25 & $21(84)$ & $4(16)$ \\
Ae. aegypti male's AGs & 26 & $0(0)$ & $26(100)$ \\
Ae. albopictus male's AGs & 37 & $1(2.7)$ & $36(97.3)$ \\
\hline Total & 88 & $22(25)$ & $66(75)$ \\
\hline
\end{tabular}


TABLE III

Number and percentage of negative and positive spermathecae of Aedes aegypti females after being exposed to Ae. aegypti and Aedes albopictus males for seven days

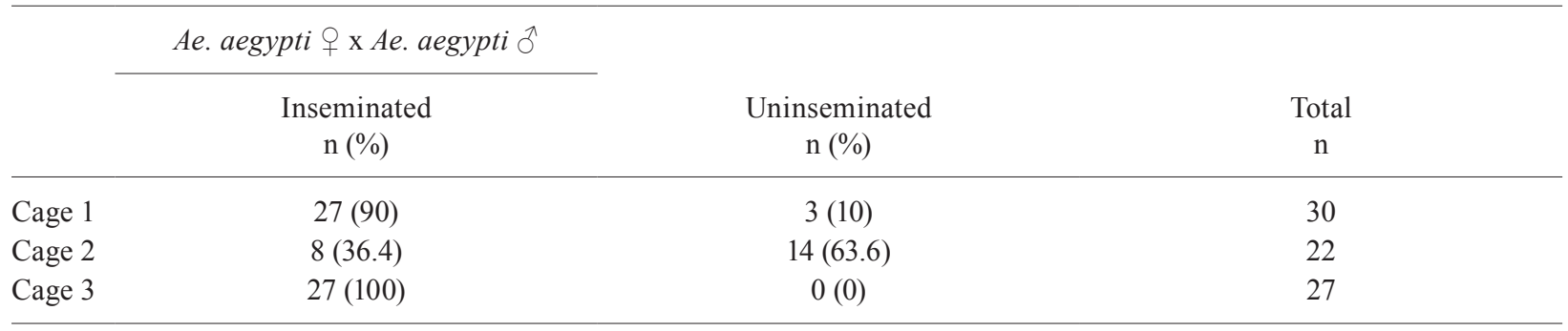

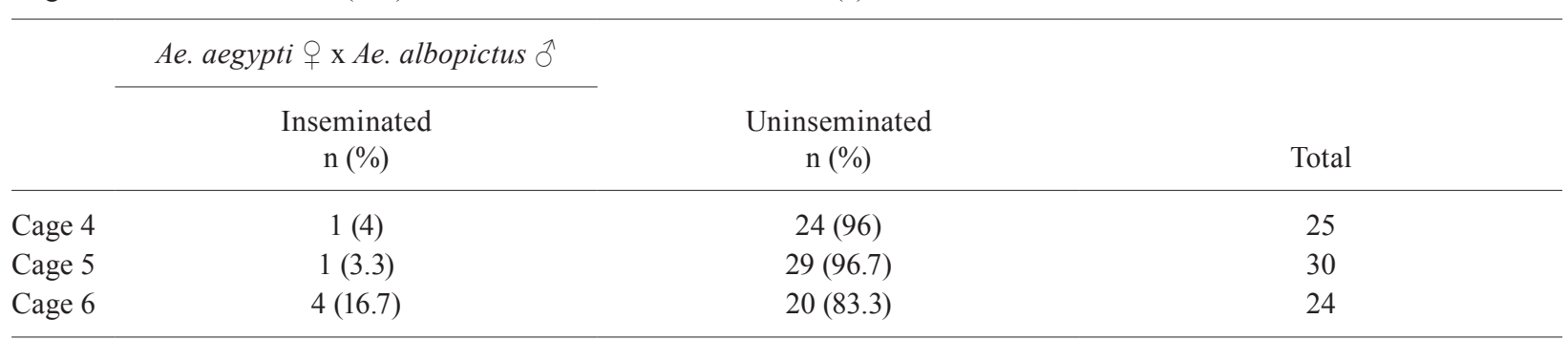

each cage previously contained 30 virgin Ae. aegypti females and 40 virgin Ae. aegypti (3 cages) or Ae. albopictus males (3 cages). All live Ae. aegypti females had their spermathecae dissected after the seven days.

experiments, we found a range of 3.3-16\% Ae. aegypti females inseminated by Ae. albopictus males. This is low compared with the conspecific mating rate $(>90 \%$ in all cages, but cage 2 with $35 \%$ insemination). It is important to point out that even with the relatively low mating frequency between Ae. aegypti females and Ae. albopictus males observed in our study (3.3-16\%) under laboratory conditions, mathematical models of satyrisation predict that parapatry or extinction can occur under conditions of $5 \%$ or less of interspecific matings (Ribeiro 1988).

Since a higher cross-mating frequency $(>30 \%)$ between Ae. aegypti females and Ae. albopictus males have been reported under laboratory conditions when adults were exposed for three weeks (Bargielowski et al. 2013), we might infer that the lower cross-mating frequency $(3.3-16 \%)$ reported in this study might be associated with the number of days Ae. aegypti females were exposed to Ae. albopictus males or cage size or previous history of the Ae. aegypti population, which is capable of evolving resistance to satyrisation (Harper \& Paulson 1994, Bargielowski et al. 2013).

Matings between wild Ae. albopictus and transgenic Ae. aegypti have been observed under laboratory conditions (Lee et al. 2009). In homologous mating between Ae. aegypti female/Ae. aegypti male and Ae. albopictus female/Ae. albopictus male, the authors reported a frequency of $87.78 \%$ and $85.56 \%$ of inseminated females, a total of 2,310 and 2,780 eggs laid and $98 \%$ and $97 \%$ of hatched eggs, respectively. Nevertheless, for reciprocal mating between Ae. aegypti female and Ae. albopictus male, there was two inseminated females (2.2\%) and 590 unviable eggs laid ( $0 \%$ hatched) (Lee et al. 2009). This insemination frequency $(2.2 \%)$ was similar with the frequencies we verified in our experiments $(3.3 \%$ and $3.6 \%)$.
Also using natural cage mating technique, Nazni et al. (2009) reported that cross mating between Ae. aegypti females and Ae. albopictus males produced more eggs than that between Ae. albopictus females and Ae. aegypti males, although none of them was viable. Similar results were reported earlier by Leahy and Craig Jr (1965).

For the first time, it was demonstrated that Ae. albopictus MAG decreases the diurnal locomotor activity of Ae. aegypti females in a similar way of Ae. aegypti MAG. In addition, this study confirmed that Ae. albopictus MAG makes Ae. aegypti females refractory to mating with conspecific males. Cross-mating between these two dengue vectors could partly explain the displacement of Ae. aegypti by Ae. albopictus and the high frequency of unviable eggs that has been observed in some areas of RJ. Moreover, we might speculate that this mating error in nature could reduce the vector capacity of an infected Ae. aegypti female by eliminating virus transmission to its offspring (vertical transmission).

\section{ACKNOWLEDGEMENTS}

To Carmen Pinheiro, NAPVE/Fiocruz (DIRAC-IOCVPAAPS), for assisting in the laboratory, and to Moacyr Silva, Getúlio Vargas Foundation, for helping in formulate mathematical models of satyrisation.

\section{REFERENCES}

Bargielowski IE, Lounibos LP, Carrasquilla MC 2013. Evolution of resistance to satyrization through reproductive character displacement in populations of invasive dengue vectors. Proc Natl Acad Sci USA 110: 2888-2892.

Bhatt S, Gething PW, Brady OJ, Messina JP, Farlow AW, Moyes CL, Drake JM, Brownstein JS, Hoen AG, Sankoh O, Myers MF, George DB, Jaenisch T, Wint GR, Simmons CP, Scott TW, Farrar JJ, Hay SI 2013. The global distribution and burden of dengue. Nature 496: 504-507. 
Braks MAH, Honório NA, Lounibos LP, Lourenço-de-Oliveira R, Juliano SA 2004. Interspecific competition between two invasive species of container mosquitoes, Aedes aegypti and Aedes albopictus (Diptera: Culicidae), in Brazil. Ann Entomol Soc Am 97: 130-139.

Braks MAH, Honório NA, Lourenço-de-Oliveira R, Juliano SA, Lounibos LP 2003. Convergent habitat segregation of Aedes aegypti and Aedes albopictus (Diptera: Culicidae) in southeastern Brazil and Florida. J Med Entomol 40: 785-794.

Brito LP, Linss JG, Lima-Camara TN, Belinato TA, Peixoto AA, Lima JB, Valle D, Martins AJ 2013. Assessing the effects of $A e$ des aegypti $\mathrm{kdr}$ mutations on pyrethroid resistance and its fitness cost. PLOS ONE 8: e60878.

Clements AN 1999. Adult circadian rhythms. In AN Clements, The biology of mosquitoes. Sensory reception and behaviour, Vol. 2, CABI publishing, London, p. 206-238.

Consoli RAGB, Lourenço-de-Oliveira R 1994. Principais mosquitos de importância sanitária no Brasil, Fiocruz, Rio de Janeiro, 225 pp.

Craig Jr GB 1967. Mosquitoes: female monogamy induced by male accessory gland substance. Science 156: 1499-1501.

Daugherty MP, Alto BW, Juliano SA 2000. Invertebrate carcasses as a resource for competing Aedes albopictus and Aedes aegypti (Diptera: Culicidae). J Med Entomol 37: 364-372.

de Castro MG, Nogueira RMR, Schatzmayr HG, Miagostovich MP, Lourenço-de-Oliveira R 2004. Dengue virus detection by using reverse transcription-polymerase chain reaction in saliva and progeny of experimentally infected Aedes albopictus from Brazil. Mem Inst Oswaldo Cruz 99: 809-814.

Degallier N, Teixeira JMS, Soares SS, Pereira RD, Pinto SCF, Chaib AJM, Vasconcelos PF, Oliveira E 2003. Aedes albopictus may not be vector of dengue virus in human epidemics in Brazil. Rev Saude Publica 37: 386-387.

Figueiredo ML, Gomes AC, Amarilla AA, Leandro AS, Orrico AS, Araujo RF, Castro JSM, Durigon EL, Aquino VH, Figueiredo LT 2010. Mosquitoes infected with dengue viruses in Brazil. Virol J 7: 152 .

Gentile C, Rivas GB, Meireles-Filho AC, Lima JB, Peixoto AA 2009. Circadian expression of clock genes in two mosquito disease vectors: cry2 is different. J Biol Rhythms 24: 444-451.

Gillott C 2003. Male accessory gland secretions: modulators of female reproductive physiology and behavior. Annu Rev Entomol 48: 163-184.

Gubler DJ, Bhattacharya NC 1972. Swarming and mating of Aedes (S.) albopictus in nature. Mosq News 32: 219-223.

Harper JP, Paulson SL 1994. Reproductive isolation between Florida strains of Aedes aegypti and Aedes albopictus. J Am Mosq Control Assoc 10: 88-92.

Hartberg WK 1971. Observations on the mating behaviour of Aedes aegypti in nature. Bull World Health Organ 45: 847-850.

Honório N, Castro MG, Barros FS, Magalhães MA, Sabroza PC 2009. The spatial distribution of Aedes aegypti and Aedes albopictus in a transition zone, Rio de Janeiro, Brazil. Cad Saude Publica 25: 1203-1214

Jones MDR 1981. The programming of circadiam flight activity in relation to mating and the gonotrophia cycle in the mosquito Aedes Aegypti. Physiol Entomol 6: 307-313.

Jones MDR, Gubbins SJ 1977. Modification of circadian flight activity in the mosquito Anopheles gambiae after insemination. $\mathrm{Na}$ ture 268: 731-732.

Jones MDR, Gubbins SJ 1978. Changes in the circadian flight activity of the mosquito Anopheles gambiae in relation to insemination, feeding and oviposition. Physiol Entomol 3: 213-220.
Juliano SA 1998. Species introduction and replacement among mosquitoes: interspecific resource competition or apparent competition? Ecology 79: 255-268.

Kaplan L, Kendell D, Robertson D, Livdahl T, Katchikian C 2010. Aedes aegypti and Aedes albopictus in Bermuda: extinction, invasion and extinction. Biol Invasions 12: 3277-3288.

Klowden MJ 1996. Endogenous factors regulating mosquito hostseeking behaviour. Ciba Found Symp 200: 212-225.

Klowden MJ 1999. The check is in the male: male mosquitoes affect female physiology and behavior. J Am Mosq Control Assoc 15: 213-220.

Leahy MG, Craig Jr GB 1965. Accessory gland substance as a stimulant for oviposition in Aedes aegypti and Ae. albopictus. Mosq News 25: 448-452.

Leahy MG, Craig Jr GB 1967. Barriers to hybridization between $A e-$ des aegypti and Aedes albopictus (Diptera: Culicidae). Evolution 21: 41-58.

Lee HL, Aramu M, Nazni WA, Selvi S, Vasan S 2009. No evidence for successful interspecific cross-mating of transgenic Aedes aegypti (L.) and wild type Aedes albopictus Skuse. Trop Biomed 26: 312-319.

Lima-Camara TN 2010. Activity patterns of Aedes aegypti and Aedes albopictus (Diptera: Culicidae) under natural and artificial conditions. Oecologia Australis 14: 737-744.

Lima-Camara TN, Bruno RV, Luz PM, Castro MG, Lourenço-deOliveira R, Sorgine MH, Peixoto AA 2011. Dengue infection increases the locomotor activity of Aedes aegypti females. PLoS ONE 6: e17690.

Lima-Camara TN, Honório NA, Lourenço-de-Oliveira R 2006. Frequência e distribuição espacial de Aedes aegypti e Aedes albopictus (Diptera:Culicidae) no Rio de Janeiro, Brasil. Cad Saude Publica 22: 2079-2084.

Lounibos LP 2002. Invasions by insect vectors of human disease. Annu Rev Entomol 47: 233-266.

Lounibos LP 2007. Competitive displacement and reduction. $J$ Am Mosq Control Assoc 23: 276-282.

Lourenço-de-Oliveira R, Vazeille M, de Filippis AMB, Failloux AB 2003. Large genetic differentiation and low variation in vector competence for dengue and yellow fever viruses of Aedes albopictus from Brazil, the United States and the Cayman Islands. Am J Trop Med Hyg 69: 105-114.

Miller BR, Ballinger ME 1988. Aedes albopictus mosquitoes introduced into Brazil: vector competence for yellow fever and dengue viruses. Trans $R$ Soc Trop Med Hyg 82: 476-477.

Nasci RS, Hare SG, Willis FS 1989. Interspecific mating between Louisiana strains of Aedes albopictus and Aedes aegypti in the field and laboratory. J Am Mosq Control Assoc 5: 416-421.

Nazni WA, Lee HL, Dayang HA, Azahari AH 2009. Cross-mating between Malaysian strains of Aedes aegypti and Aedes albopictus in the laboratory. Southeast Asian J Trop Med Public Health 40: 40-46.

R Core Team 2012. R: a language and environment for statistical computing, R Foundation for Statistical Computing, Vienna. Available from: R-project.org/.

Rey JR, Nishimura N, Wagner B, Braks MA, O'Connell SM, Lounibos LP 2006. Habitat segregation of mosquito arbovirus vectors in south Florida. J Med Entomol 43: 1134-1141.

Ribeiro JM 1988. Can satyrs control pests and vectors? J Med Entomol 25: 431-440.

Ribeiro JMC, Spielman A 1986. The satyr effect: a model predicting parapatry and species extinction. Am Nat 128: 513-528. 
Rowland M 1989. Changes in the circadian flight activity of the mosquito Anopheles stephensi associated with insemination, bloodfeeding, oviposition and nocturnal light intensity. Physiol Entomol 14: 77-84.

Serufo JC, de Oca HM, Tavares VA, Souza AM, Rosa RV, Jamal MC, Lemos JR, Oliveira MA, Nogueira RMR, Schatzmayr HG 1993. Isolation of dengue virus type 1 from larvae of Aedes albopictus in Campos Altos city, state of Minas Gerais, Brazil. Mem Inst Oswaldo Cruz 88: 503-504.

Shutt B, Stables L, Aboagye-Antwi F, Moran J, Tripet F 2010. Male accessory gland proteins induce female monogamy in anopheline mosquitoes. Med Vet Entomol 24: 91-94.
Sirot LK, Poulson RL, McKenna MC, Girnary H, Wolfner MF, Harrington LC 2008. Identity and transfer of male reproductive gland proteins of the dengue vector mosquito, Aedes aegypti: potential tools for control of female feeding and reproduction. Insect Biochem Mol Biol 38: 176-189.

Tripet F, Lounibos LP, Robbins D, Moran J, Nishimura N, Blosser EM 2011. Competitive reduction by satyrization? Evidence for interspecific mating in nature and asymmetric reproductive competition between invasive mosquito vectors. Am J Trop Med Hyg 85: $265-270$.

Williams CB 1937. The use of logarithms in the interpretation of certain entomological problems. Ann Appl Biol 24: 404-414. 